\title{
Unraveling human natural killer cell deficiency
}

\author{
Jordan S. Orange \\ University of Pennsylvania School of Medicine, Department of Pediatrics, Children's Hospital of Philadelphia Research Institute, \\ Philadelphia, Pennsylvania, USA.
}

\begin{abstract}
NK cells are a component of the innate immune system identified in animals as serving an essential role in antiviral immunity. Establishing their role in human health has been challenging, with the most direct insight coming from the study of NK cell-deficient individuals. However, NK cell deficiencies are rare, and more research is needed. In this issue of the JCI, two independent groups of researchers have simultaneously identified the genetic cause of a human NK cell deficiency as mutation in the MCM4 gene, encoding minichromosome maintenance complex component 4 . These reports suggest a critical role for the minichromosome maintenance helicase complex in NK cells and NK cell-mediated host defense.
\end{abstract}

\section{NK cells and their deficiency in humans}

NK cells are lymphocytes of the innate immune system that serve essential roles in host defense (1). They are specialized for surveillance and are alerted to particular signatures of disease through a series of germline-encoded activating and inhibitory receptors. NK cell functions known to participate in immunity include the secretion of cytokines, costimulation of other immune cells, and contact-dependent cytotoxicity of targeted cells. The latter occurs through the directed secretion of the contents of highly specialized lysosome-related organelles (which are called lytic granules) that include the pore-forming molecule perforin and apoptosis-inducing proteases (2).

While there is abundant mechanistic evidence in experimental animals regarding the important role of NK cells in immune defense (3), similar proof in humans has been more difficult to obtain. Some of the most valuable evidence has been generated from the study of very rare NK cell-deficient patients $(4,5)$. Like NK cell-deficient animals, these individuals are extraordinarily susceptible to infection with viruses, particularly those of the herpesvirus and papillomavirus families. Both of these virus families have well-developed strategies for evading cytotoxic $\mathrm{T}$ cell responses, perhaps making NK cells a more relevant feature of antiviral defense. There are likely other factors, however, as other viruses that evade

Conflict of interest: The author has declared that no conflict of interest exists.

Citation for this article: J Clin Invest. 2012; 122(3):798-801. doi:10.1172/JCI62620. cytotoxic $\mathrm{T}$ cells (6) are not as burdensome in patients with NK cell deficiency.

Human NK cell deficiencies can be divided into two categories. Those in the first category are characterized by effects on the quantity of NK cells in the peripheral blood, while effects on the function but not quantity of NK cells characterize those in the second. NK cell deficiencies in the first category have been labeled "classical NK cell deficiencies", and those in the second "functional NK cell deficiencies" $(4,5)$. The first example of a classical NK cell deficiency was reported in 1989 in a girl with severe varicella and other complicated herpesvirus infections (7). She was determined to stably lack both NK cells in peripheral blood as well as peripheral blood NK cell cytotoxic activity against the prototypical human NK cell target cell, the K562 erythroleukemia cell line. The first example of a functional NK cell deficiency was described in 1982 in three siblings with severe Epstein-Barr virus infection (8). All three individuals in this family had deficient K562 target cell killing activity, and the surviving affected individual has had persistently deficient function over a 30-year period (personal observation).

Although reports of individuals with these rare disorders have accumulated, the mechanistic underpinnings of the conditions remain largely unknown. Only two genetic causes of NK cell deficiency have been identified to date. These are autosomal recessive $C D 16$ mutation, which leads to a functional NK cell deficiency (9), and autosomal dominant GATA2 mutation, which leads to classical NK cell deficiency $(10,11)$. Individuals with either of these mutations have characteristic viral suscep- tibility, although some individuals with GATA2 mutations have abnormalities in monocytes and dendritic cells in addition to NK cells and are also susceptible to atypical mycobacterial infection. It is presently unclear why autosomal recessive $C D 16$ mutation and autosomal dominant GATA2 mutation affect NK cells.

When the experience obtained from studying the rare cases of classical and functional NK cell deficiency is combined with that gained from the analysis of other known human single gene diseases that impact the immune system more broadly but include deficiency in NK cell numbers or function $(4,5)$, some fairly substantive insights can be obtained into the role of human NK cells in host defense as well as their requirements for existence and function (Figure 1). Even though some of these diseases are quite immunologically severe, most are associated with abnormal susceptibility to herpesviruses in at least some form. Thus, while immunologically instructive, they have not pointed to any NK cell-specific developmental requirements or a direct clinical role for NK cells in the way the classical or functional NK cell deficiencies have.

\section{MCM4 mutation is a novel cause of NK cell deficiency and adrenal insufficiency}

In this issue of the JCI, Gineau et al. (12) and Hughes et al. (13) have defined one of the clearest known examples of classical NK cell deficiency as resulting from mutations in the minichromosome maintenance complex component 4 (MCM4; also known as minichromosome maintenance-deficient 4) gene. The patients are from three consanguineous Irish cohorts and in addition to exhibiting classical NK cell deficiency have adrenal insufficiency and growth retardation. From an infectious standpoint, the proband of the first family studied experienced complications from EBV infection and other affected individuals had susceptibility to infection with herpesviruses and presumed complications of viral illnesses - symptoms clinically consistent with an NK cell deficiency. 


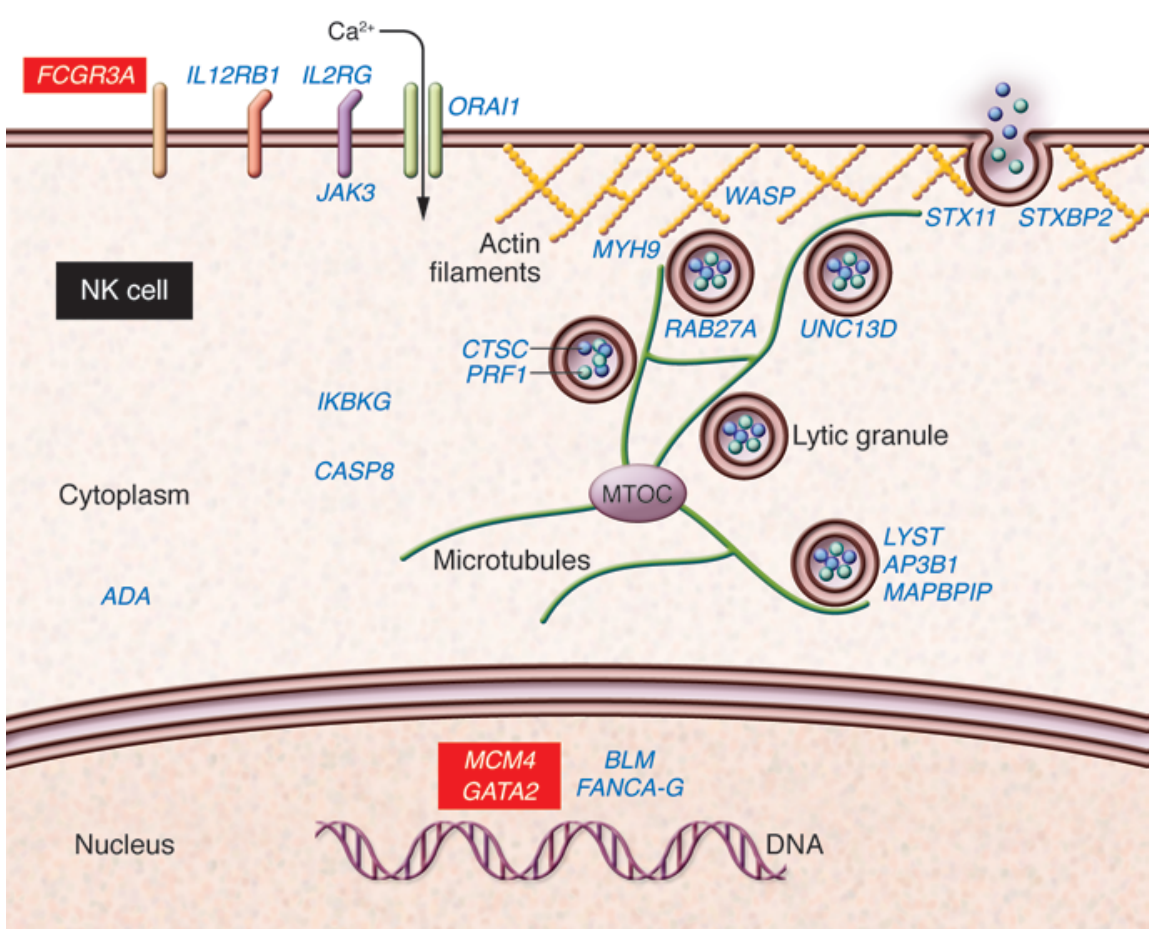

\section{Figure 1}

Human genes known to affect NK cells in the context of naturally occurring disease. A human NK cell is represented schematically, with disease-associated genes listed in proximity to where the encoded proteins function within the cell. Genes that have an impact on other immune cells in addition to NK cells and thus cause broader immunodeficiency/hematological syndromes are shown in blue. The three genes known to cause either functional (CD16) or classical (GATA2 and now MCM4) NK cell deficiency are shown in red. CD16 mutation impacts a region of the well-defined NK cell activation receptor (shown on the cell surface) encoded by the gene (9), while GATA2 mutation affects the broadly expressed hematopoietic transcription factor (shown in the nucleus) that it encodes $(10,11)$. MCM4 mutations as defined by Gineau et al. (12) and Hughes et al. (13) presumably affect the function in NK cell DNA replication of the MCM2-7 complex of which MCM4 is a component. The impact and role in NK cells of the other human disease-associated mutated genes is reviewed elsewhere $(2,4,5)$. In addition to having an impact on other immune cells, these affect NK cell development (IL2RG, JAK3), NK cell survival (ADA, BLM, FANCA-G), NK cell lytic function (ITGB2, ORAI1, LYST, AP3B1, MAPBPIP, RAB27A, UNC13D, STXBP2, STX11, PRF1, CTSC, WASP, MYH9), or NK cell responsiveness (TAP1, TAP2, IL12RB1, NEMO, CASP8).

Several of the patients studied have been known to have an NK cell deficiency for quite some time (14); colleagues of Gineau have previously identified low numbers of peripheral blood NK cells in the four affected children of the larger family studied (14). Using microsatellite homozygosity mapping, this group had linked the phenotype to chromosome $8 p 11.23$-q11.21 with a lod score of $4.51(14)$. This is a $12-\mathrm{Mb}$ region containing 59 genes, which were subsequently focused to 45 and systematically evaluated by Gineau et al. (12). Colleagues of Hughes had originally observed and studied the adrenal insufficiency exhibited by affected individuals in one of the cohorts (15). In the present study,
Hughes et al. discovered the causative gene via genome-wide homozygosity mapping and a targeted custom exonic sequence capture array (13). Both groups identified the causative mutations as homozygous acceptor splice site mutations in the MCM4 gene leading to a frameshift and premature stop codon $(12,13)$. This results in a severely truncated MCM4 protein and abrogation of the major MCM4 isoform.

\section{MCM4 uniquely impacts NK cell biology}

MCM4 is one part of the minichromosome maintenance complex consisting of MCM2-7. It is a highly conserved DNA helicase that is recruited to origins of rep- lication to promote the unwinding and polymerization of chromosomal DNA (16). Complete MCM4 deficiency in mice is lethal, although a hypomorphic mutant mouse strain (Chaos3) has been characterized (17). In the mutations identified in patients with NK cell deficiency, adrenal insufficiency, and growth retardation, Gineau et al. found that the MCM complex formed normally but failed to promote normal DNA replication, resulting in an abnormal proportion of cells in $\mathrm{G}_{1} / \mathrm{S}$ phase (12). In patient-derived fibroblasts there was also an abnormally high number of DNA breaks, thus confirming aberrant replicative processes.

Why an impact upon the seemingly ubiquitous and essential MCM2-7 complex and inclusion of an aberrant MCM4 component would affect only NK cells, adrenal function, and growth patterns is unclear. Although direct patient samples were not available, in Chaos 3 mice, Hughes et al. found that adrenal morphology was abnormal, with displacement of steroidogenic cells. This potentially would impact normal adrenal steroid responses (13).

The NK cell phenotype in the patients is especially unusual. While the total number of NK cells in their peripheral blood is extremely low, there is a normal number of CD56 $6^{\text {bright }} \mathrm{NK}$ cells. CD56 $6^{\text {bright }} \mathrm{NK}$ cells are believed to be immature and a developmental precursor to CD56 dim mature NK cells (Figure 2 and ref. 18). In peripheral blood, CD56 $6^{\text {bright }} \mathrm{NK}$ cells represent a minor population of NK cells, typically approximately $5 \%$. They have characteristic cell surface receptor phenotypes and are not enabled for cytotoxicity. Although the exact requirements are unclear, $\mathrm{CD} 56^{\text {bright }} \mathrm{NK}$ cells are thought to give rise to CD56 $6^{\mathrm{dim}} \mathrm{NK}$ cells in peripheral lymphoid organs and thus sustain the population of cytotoxic NK cells essential for host defense $(18,19)$. In their report, Gineau et al. observed a selective defect in the proliferation of mutationcontaining CD56 $6^{\text {bright }} \mathrm{NK}$ cells as well as an increase in their spontaneous apoptosis (12). This defect was not identified in other immune cell populations tested.

The observations of Gineau et al. (12) underscore the importance of mature CD56 dim lytic NK cells in human host defense and suggest that MCM4 is required for terminal NK cell maturation. Specific evaluation of the patients' lymphoid compartments, where $\mathrm{CD} 56^{\text {bright }}$ cells are believed to undergo maturation, as well as the recapitulation of NK cell development 


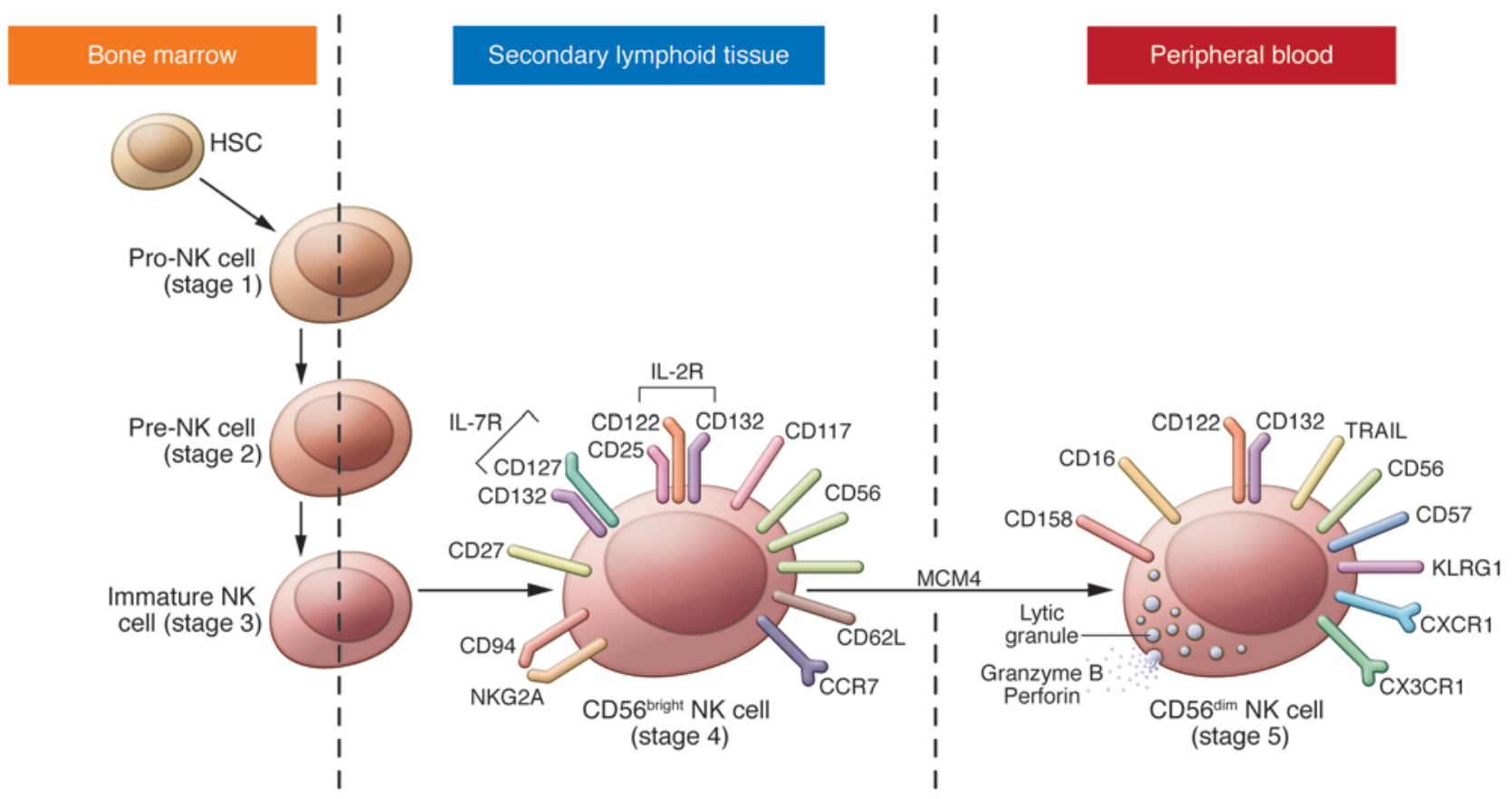

Figure 2

Schematic of terminal human NK cell differentiation and a proposed role for MCM4. Human NK cells arise from the HSC in the bone marrow and then progress to pro-, pre-, and immature NK cells (also referred to as stage 1, 2, and 3 NK cells, respectively). The immature NK cell transits to the peripheral lymphoid compartment secondary lymphoid tissues where CD56 bright NK cells predominate. CD56 bright NK cells are also referred to as stage $4 \mathrm{NK}$ cells, and current evidence suggests that they develop in the secondary lymphoid tissues into CD56 dim mature NK cells (stage 5). Key differences between CD56 bright and CD56 dim NK cells are depicted in the schematics of these two cells. Characteristics of early-stage NK cells as well as their specific transcriptional requirements are reviewed elsewhere (18). The present work suggests a role for MCM4 in the transition of CD56 bright to CD56 dim NK cells, as evidenced by the relative absence of CD56dim NK cells in the peripheral blood with the preservation of the CD56 bright NK cell population.

in vitro from patient cells would provide insight. Furthermore, the question arises as to a potential role for MCM4 in the survival of mature NK cells as opposed to a defect in development or transition from CD56 6 bright NK cells. Additional biological studies of patient cells as well as NK cells in which MCM4 has been experimentally manipulated should prove mechanistically useful.

\section{A new disease mechanism and new horizons in NK cell biology}

Collectively, the works of Gineau et al. (12) and Hughes et al. (13) provide deep mechanistic perspective into a novel human disease phenotype and directly expand our understanding of human immunobiology and endocrinology. A clear outstanding question, however, remains: why such selectivity for NK cells and adrenal steroidogenic cells? This unexpected discovery suggests the need to better understand the specific replicative requirements of immature NK cells and adrenal cells and why these cells are unique within the immune and endocrine systems, respectively, in their reliance upon MCM4. Since deficiencies of NK cells have been identified in other DNA stability defects (reviewed in ref. 4), there is likely to be some particular importance of specific types of DNA replication in NK cells and their development.

There are also certain other features of the reports from Gineau et al. (12) and Hughes et al. (13) that warrant further consideration. The total peripheral blood NK cell counts reported by Hughes et al. (13) were not as low as those observed by Gineau et al. (12), and in some affected individuals approached normal ranges. This raises the possibility that this particular mutant MCM4-associated phenotype shows variable expressivity. Penetrance, however, would appear to be complete, as all individuals had low numbers of NK cells. It is unclear whether this is simply something that changes over time in affected individuals or is a feature of genetic co-modifiers in these consanguineous cohorts, or even environmental experience and/or epigenetic modifications. Whichever the case, it may help explain the observation that only some of the patients were affected by the profound susceptibility to viral infections observed in the proband.

It is likely that defects in genes encoding products with a functional relationship with MCM4 will be defined in other reported classical and functional NK cell deficiencies, since certain cases described in the literature are characterized by clinical and immunologic phenotypes strikingly similar to those reported by Gineau et al. (12) and Hughes et al. (13) (for example, it has been reported that individuals with classical NK cell deficiency in a non-consanguineous French family also exhibit growth retardation, although an impact on neutrophils and cytotoxic T cells is also seen; ref. 20). Nevertheless, the work of Gineau et al. (12) and Hughes et al. (13) defines a new genetic syndrome that establishes the importance of mature NK cells in human host defense and suggests novel molecular requirements in NK cell maturation. The further definition of human NK cell deficiency and its genetic roots also define the clinical relevance of this diagno- 
sis and the need for continued research if we are to develop therapeutics that benefit affected patients.

\section{Acknowledgments}

I thank Emily Mace for the critical review of this commentary, as well as the support of NIH-NIAID R01067946.

Address correspondence to: Jordan S. Orange, Children's Hospital of Philadelphia Research Institute, 3615 Civic Center Blvd., ARC 907A, Philadelphia, Pennsylvania 19104, USA. Phone 267.426.5622, Fax 267.426.0947; E-mail: orange@upenn.edu.

1. Sun JC, Lanier LL. NK cell development, homeostasis and function: parallels with CD8 T cells. Nat Rev Immunol. 2011;11(10):645-657.

2. Orange JS. Formation and function of the lytic NK-cell immunological synapse. Nat Rev Immunol. 2008;8(9):713-725.

3. Lee SH, Biron CA. Here today - not gone tomorrow: roles for activating receptors in sustaining NK cells during viral infections. Eur J Immunol.
2010;40(4):923-932.

4. Orange JS. Human natural killer cell deficiencies and susceptibility to infection. Microbes Infect. 2002;4(15):1545-1558.

5 . Orange JS. Human natural killer cell deficiencies. Curr Opin Allergy Clin Immunol. 2006;6(6):399-409.

6. Tortorella D, Gewurz BE, Furman MH, Schust DJ, Ploegh HL. Viral subversion of the immune system. Annu Rev Immunol. 2000;18:861-926.

7. Biron CA, Byron KS, Sullivan JL. Severe herpesvirus infections in an adolescent without natural killer cells. N Engl J Med. 1989;320(26):1731-1735.

8. Fleisher G, Starr S, Koven N, Kamiya H, Douglas $\mathrm{SD}$, Henle W. A non-x-linked syndrome with susceptibility to severe Epstein-Barr virus infections. J Pediatr. 1982;100(5):727-730.

9. Jawahar S, Moody C, Chan M, Finberg R, Geha R, Chatila T. Natural Killer (NK) cell deficiency associated with an epitope-deficient Fc receptor type IIIA (CD16-II). Clin Exp Immunol. 1996;103(3):408-413.

10. Dickinson RE, et al. Exome sequencing identifies GATA-2 mutation as the cause of dendritic cell, monocyte, B and NK lymphoid deficiency. Blood. 2011;118(10):2656-2658.

11. Hsu AP, et al. Mutations in GATA2 are associated with the autosomal dominant and sporadic monocytopenia and mycobacterial infection (MonoMAC) syndrome. Blood. 2011;118(10):2653-2655.

12. Gineau L, et al. Partial MCM4 deficiency in patients with growth retardation, adrenal insufficiency, and natural killer cell deficiency. J Clin Invest. 2012; 122(3):821-832.

13. Hughes CR, et al. MCM4 mutation causes adrenal failure, short stature, and natural killer cell deficiency in humans. J Clin Invest. 2012;122(3):814-820.

14. Eidenschenk C, et al. A novel primary immunodeficiency with specific natural-killer cell deficiency maps to the centromeric region of chromosome 8 . Am J Hum Genet. 2006;78(4):721-727.

15. O'Riordan SM, Lynch SA, Hindmarsh PC, Chan LF, Clark AJ, Costigan C. A novel variant of familial glucocorticoid deficiency prevalent among the Irish Traveler population. J Clin Endocrinol Metab. 2008;93(7):2896-2899.

16. Bochman ML, Schwacha A. The Mcm complex: unwinding the mechanism of a replicative helicase. Microbiol Mol Biol Rev. 2009;73(4):652-683.

17. Shima N, et al. A viable allele of $\mathrm{Mcm} 4$ causes chromosome instability and mammary adenocarcinomas in mice. Nat Genet. 2007;39(1):93-98.

18. Freud AG, Caligiuri MA. Human natural killer cell development. Immunol Rev. 2006;214:56-72.

19. Cooley S, et al. A subpopulation of human peripheral blood NK cells that lacks inhibitory receptors for self-MHC is developmentally immature. Blood. 2007;110(2):578-586.

20. Bernard F, et al. A novel developmental and immunodeficiency syndrome associated with intrauterine growth retardation and a lack of natural killer cells. Pediatrics. 2004;113(1 pt 1):136-141

\section{Unraveling the mystery of the hygiene hypothesis through Helicobacter pylori infection}

Kouji Matsushima ${ }^{1}$ and Shigenori Nagai ${ }^{2}$

${ }^{1}$ Department of Molecular Preventive Medicine, Graduate School of Medicine, The University of Tokyo, Tokyo, Japan.

2Department of Microbiology and Immunology, Keio University School of Medicine, Tokyo, Japan.

\begin{abstract}
Epidemiological studies have revealed an inverse association between $\mathrm{Heli}$ cobacter pylori infection and the incidence of allergic asthma. This association is consistent with the hygiene hypothesis, which posits that exposure to microbes early in life prevents the later development of allergic diseases, and has been reproduced in mouse models of asthma. In this issue of the $J C I$, Oertli and colleagues report that $H$. pylori infection in neonates elicits tolerogenic DCs that produce IL-18, which drive the generation of Tregs that subsequently protect the mice from allergic asthma. This finding strengthens the intriguing link between pathogen exposure and allergic disease.
\end{abstract}

Nearly half of the world's population is infected with the Gram-negative bacterium Helicobacter pylori. Infection rates are higher in developing countries than in developed countries; in fact, the bacterium is gradually disappearing from many populations in developed countries (1). Infection seems to occur in early childhood in most cases, with the incidence of infection increasing

Conflict of interest: The authors have declared that no conflict of interest exists.

Citation for this article: J Clin Invest. 2012;

122(3):801-804. doi:10.1172/JCI61466. with age. German scientists first observed the spiral-shaped bacterium in the lining of the human stomach in 1875 (2). However, Australian scientists, Marshall and Warren, were the first to culture this bacterium in 1984, and they went on to link the presence of $H$. pylori in the gut to inflammation in the stomach (gastritis) and ulceration of the stomach or duodenum (peptic ulcer disease) (3). Marshall and Warren were awarded the 2005 Nobel Prize in Physiology or Medicine for this finding. H. pylori infection has been subsequently associated with gastric cancer, mucosa-associated tis- sue lymphoma, gastroesophageal reflux disease, and iron deficiency anemia (4).

The flip side of the equation is that, in an epidemiological study, Chen and Blaser reported an inverse association between infection with $H$. pylori expressing the virulence factor cytotoxin-associated protein $\mathrm{A}$ (CagA) and the incidence of asthma and allergy (5). This observation is consistent with the hygiene hypothesis, which states that exposure to microbes (both pathogenic and commensal) early in life prevents the later development of allergic diseases (6). The basis for this phenomenon is unclear, but, recently, Müller and colleagues demonstrated that $H$. pylori infection prevents allergic asthma in mouse models of the condition through the induction of Tregs (7).

The logical question raised by this discovery was what is the mechanism underlying the induction of Tregs in mice infected with H. pylori? In this issue of the JCI, Oertli, Müller, and colleagues provide insight into this process (8). Specifically, they show that 